Recibido/received: 03 agosto 2015 | Aceptado/accepted: 18 noviembre 2015

\title{
Evaluación personal de las estrategias durante el proceso de la comprensión lectora. Esencia en la formación profesional
}

Mirtha Manzano Díaz; Eugenio Hidalgo Diez² Alina Morales Chávez³, Mabel Acosta García

Universidad Estatal de Bolívar

Universidad de Granada, España²

Colegio Gonzalo Zaldumbide, Quito, Ecuador ${ }^{3}$

mansanolebroc@gmail.com

\section{Resumen}

Las estrategias de lectura para el aprendizaje, durante la formación profesional son herramientas esenciales, ellas pueden transmitirse y entrenarse a través del proceso docente-educativo en las diferentes disciplinas y carreras en la educación superior. Por ello, hemos elaborado un cuestionario que analiza los procesos cognitivos y afectivos en relación con la comprensión lectora a diferentes niveles, donde se asumieron dimensiones tales como: Conocimiento previo y metacognición, nivel de traducción, nivel de interpretación y nivel de extrapolación. Se aplicó el juicio de expertos y el análisis factorial para confirmar su validez y se estableció el Alpha de Cronbach para definir su fiabilidad; los resultados de los estadísticos corroboraron la bondad del cuestionario, lo que nos permitirá, evaluar, autoevaluar el uso de estrategias de lectura y sistematizarlas durante el proceso de enseñanza-aprendizaje.

Palabras claves: comprensión de lectura, estrategias de lectura, evaluación.

\begin{abstract}
Learning strategies in the reading comprehension process during the future professionals' formation are significant tools. They may be transmitted and trained through the different disciplines during the teaching-learning process in in all the majors of Higher Education. For this reason, the instrument we have elaborated is aimed at evaluating the students' own cognitive and affective procedures through the reading comprehension process at different levels. Therefore, after an exhaustive bibliographical review, four categories with their corresponding items were established: previous knowledge and metacognition, translation level, interpretation level, and extrapolation level. Finally, to confirm its validity and to validate the reliability of the questionnaire we used methods, such as, criterion of experts, factorial analysis, and Alpha of Cronbach; their results confirmed the feasibility of the questionnaire to evaluate the use of reading strategies and systematize them during the teaching-learning process.
\end{abstract}

Keywords: reading comprehension, reading strategies, evaluation.

Cómo citar este artículo: Manzano M., Hidalgo E., Morales A. y Acosta M. (2015) Evaluación personal de las estrategias durante el proceso de la comprensión lectora. Esencia en la formación profesional. CUMBRES, Revista Científica. 1(2) 16 - 21

\section{Cumbres}




\section{Introducción}

Aunque los profesores de lengua, generalmente hablamos en términos de habilidades lingüísticas o comunicativas (expresión oral y escrita y comprensión auditiva y de lectura), la realidad es que no siempre somos conscientes de que a través de ellas se desarrollan otras habilidades intelectuales y científicas y de que se establecen valores necesarios para el graduado de cualquier campo del saber, por eso, actualmente, se incluyen en los planes de estudio como competencias que se precisan para la formación de un profesional. Estas competencias determinan el verdadero propósito de la comunicación y la actividad cognoscitiva (Hidalgo, 2007). Nuestra investigación está dirigida a la comprensión de la lectura, pero sustentada sobre la base de que forma parte de la lengua como una entidad, pues el lenguaje, aunque metodológicamente lo separemos para su estudio, no es sólo palabra unida, el lenguaje es sentido, es intención y tiene, además de su carácter universal, su propia identidad, por lo tanto, también es contexto.

Nuestro estudio asume una posición compleja y sistémica del proceso de enseñanza aprendizaje de una lengua extranjera, por lo que entendemos, que las limitaciones que confrontamos en la propia concepción de la enseñanza-aprendizaje de la lengua como un todo, son aplicables, también al desarrollo de la competencia de lectura como parte esencial de la competencia comunicativa. En este sentido, consideramos que la lectura es, además de objetivo y contenido, medio, método y evaluación en el proceso de enseñanza-aprendizaje. Esta concepción de la lectura como instrumento didáctico de los aspectos lingüísticos, y como herramienta que desarrolla y forma en la sistematización de los contenidos, y también en la adquisición de una cultura integral, es la que ha frenado la formación adecuada en la competencia comunicativa a nuestros futuros profesionales, dando lugar a que sólo aquellos con una elevada motivación e independencia cognoscitiva alcancen los mejores resultados en la formación académica en una lengua extranjera. (Ascuy, et al. 2007; Manzano, 2007)

Por todo ello, el artículo presenta todo el proceso seguido en la elaboración y validación de un cuestionario de evaluación-autoevaluación de estrategias de lectura, partiendo, para ser congruente con nuestra posición epistemológica, de la significación que adquiere la evaluación y concluye con la dinámica que promueve este instrumento como medio y método de enseñanza-aprendizaje, durante el proceso de comprensión en una lengua extranjera.

\section{Análisis-Disertación}

Cuando hablamos del proceso de enseñanza-aprendizaje de una lengua extranjera nos estamos refiriendo a la actividad cognoscitiva, cuando nos referimos a las habilidades de la actividad verbal (lingüísticas), estamos haciendo referencia a la expresión y comprensión oral y escrita, a las que también denominamos destrezas comunicativas. Si vamos a la esencia de esas acciones (destrezas o habilidades), no podemos obviar los procesos mentales que durante las mismas ocurren, ni obviar operaciones o estrategias que el ser humano necesita para lograr el objetivo de determinada tarea, en las que intervienen las esferas motivacional-afectiva y cognitivo-instrumental. (Leontiev, 1982; Maciel, 2005)

La evaluación-autoevaluación es condición esencial en el aprendizaje, la misma regularmente se asume como un elemento externo que influye desde la visión y posición del profesor como director del proceso, es un error soslayar sus potencialidades como condición interna. La evaluación-autoevaluación es motor que activa de forma consciente el aprendizaje y será cualitativamente superior en tanto esté concebida de tal forma que contribuya a la autonomía del alumnado y que valore cómo aprender. (Gil y Padilla, 2009; González, 2009; Milán, et al. 2009)

Finalmente, indicar, teniendo en cuenta a Fitzpatrick, (2006), Brodie e Irving, (2007) y Gil y Padilla (2009) que la participación del alumnado en el proceso evaluativo es imprescindible, ya que desarrolla el pensamiento analítico, el espíritu crítico, la emisión de juicios y criterios y la autodeterminación. Así pues, la evaluación se convierte en un elemento fundamental e inseparable de la práctica educativa, y permite dar, al estudiante y profesorado, la información de cada momento y realizar los juicios de valor necesarios para la orientación y toma de decisiones respecto al proceso de enseñanza-aprendizaje (Hidalgo, 1996). Estas habilidades están en congruencia con el propio desarrollo de las estrategias de lectura, ya que forman parte de los indicadores seleccionados en el cuestionario de evaluación. Al seleccionar el Cuestionario como instrumento para evaluar el uso de estrategias de lectura, se hace necesario fundamentar teóricamente las dimensiones e indicadores asumidos en su elaboración, lo que vendrá a fortalecer con elementos teóricos sustanciales, su validez y fiabilidad.

Descripción de los procedimientos en la elaboración del cuestionario de evaluación de las estrategias de lectura. El cuestionario de evaluación-autoevaluación de 
estrategias de lectura, constituye una nueva aportación en este campo. Para su elaboración se siguió el siguiente proceso:

Se delimitó, como campo de estudio, el proceso de lectura y dentro de su complejidad, se seleccionaron diferentes acciones y operaciones o estrategias a niveles de traducción, interpretación y extrapolación, que respondieran a los propósitos esenciales de nuestros programas y planes de estudio. También, se seleccionaron operaciones dirigidas a establecer los metaconocimientos de los procesos mentales del individuo.

Se realizó una lista de indicadores y categorías que cubrieran la esencia de las estrategias de lectura en correspondencia con las dimensiones. (Tabla 1)

Se seleccionaron para cada uno de los indicadores tres ítems o más que respondieran a lo que se proponía medir utilizando una escala Lickert con seis opciones, quedando conformado el cuerpo esencial del instrumento. También se definió la forma de evaluación para facilitarle los resultados al propio alumnado.

- Se aplicaron métodos empíricos y estadísticos para establecer las características técnicas de fiabilidad y validez de contenido y de constructo del cuestionario de evaluación de las estrategias de lectura.

Tabla 1: Resumen de las dimensiones e indicadores seleccionados

\begin{tabular}{|c|c|c|}
\hline DIMENSIONES & INDICADORES & ITEMS \\
\hline $\begin{array}{c}\text { A- } \\
\text { CONOCIMIENT } \\
\text { O PREVIO }\end{array}$ & $\begin{array}{l}\text { - Trazarse un propósito para la lectura } \\
\text {-Activar los conocimientos previos } \\
\text {-Tener conciencia de los propios procesos de } \\
\text { pensamiento }\end{array}$ & $1-13$ \\
\hline $\begin{array}{l}\text { B- NIVEL DE } \\
\text { TRADUCCIÓN }\end{array}$ & $\begin{array}{l}\text { - Comprender globalmente } \\
\text {-Reconocer significados: explícitos, implícitos y } \\
\text { complementarios } \\
\text { - Reconocer la estructura textual } \\
\text {-Organizar, identificar y jerarquizar ideas } \\
\text {-Resumir }\end{array}$ & $14-39$ \\
\hline $\begin{array}{c}\text { C- NIVEL DE } \\
\text { INTERPRETACI } \\
\text { ÓN }\end{array}$ & $\begin{array}{l}\text {-Inferir información implícita } \\
\text {-Valorar la información } \\
\text {-Emitir juicios y criterios }\end{array}$ & $40-52$ \\
\hline $\begin{array}{c}\text { D-NIVEL DE } \\
\text { EXTRAPOLACI } \\
\text { ÓN }\end{array}$ & $\begin{array}{l}\text {-Extender la información } \\
\text {-Analizar situaciones similares } \\
\text {-Resolver problemas }\end{array}$ & $52-66$ \\
\hline
\end{tabular}

Una vez establecidas las dimensiones y los indicadores correspondientes, se procedió a la definición de los diferentes ítems que iban a configurar el cuestionario, quedando, definitivamente elaborado. (Consultar cuestionario completo en: http://dialnet.unirioja.es/servlet/articulo?codigo=4543230).

\section{Características técnicas del cuestionario de estrate-} gias de lectura: su fiabilidad y validez. La validez y la fiabilidad son constantes que deben estar siempre presentes en el proceso de elaboración de un cuestionario, sobre todo, en nuestro caso, en el que se miden operaciones que realiza el individuo, generalmente, de forma inconsciente, como las estrategias de aprendizaje, por todo ello, se hace necesario ser cuidadoso y riguroso en el proceso de elaboración y de validación.

Análisis y Estudio de la validez de contenido: En principio, se distribuyó el Cuestionario preparado para facilitar su análisis a los jueces que actuaron como expertos, entre los que figuraban: Profesorado del Departamento de Métodos de Investigación y Diagnóstico en Educación, del Departamento de Lengua Extrajera y Literatura y del Departamento de Psicología Evolutiva y de la Educación, así como personas de otros estamentos tales como: Asesores de los Centros de Profesorado e Inspectores de los Servicios Técnicos de Inspección. Los jueces debían seleccionar de entre una escala de 4 opciones: 1=Nada; 2=Poco; 3=Bastante; 4=Totalmente. Valorando, con ello, el grado de relación entre los ítems y las dimensiones establecidas.

La valoración dada por los diez jueces expertos quedó reflejada entre los rangos: bastante y totalmente. Con respecto a su univocidad y pertinencia todos estuvieron de acuerdo en que los ítems eran claros, concisos, concretos, se entendían bien y eran pertinentes para el estudio. Estos resultados, finalmente, confirman que los expertos concuerdan en que las preguntas contenidas dentro del cuestionario diseñado, corresponden al objetivo esencial de nuestro instrumento y de nuestro estudio, lo que permite corroborar su validez de contenido.

Análisis y estudio de la validez de constructo: El análisis factorial es aconsejable para estudiar la estructura de los cuestionarios de manera que podamos afirmar que determinados ítems se explican mejor desde una dimensión que desde otra, incluso, nos sugiere aquellos ítems que puedan ser eliminados por escasa consistencia interna en correspondencia con otros de las diferentes dimensiones seleccionadas. (García, Gil y Rodríguez, 2000; Hidalgo, 2003)

\section{Cumbres}


Para lo que realizamos el análisis factorial, obteniendo los siguientes resultados:

Matriz de correlaciones. Los determinantes de la matriz fueron bajos, lo que confirmó que las correlaciones no son debidas al azar. Demostrando, así, la intercorrelación entre las dimensiones del instrumento.

La prueba de esfericidad de Bartlett. Probó la no significatividad de la hipótesis nula de que la matriz de correlaciones era una matriz de identidad y que por lo tanto tiene sentido aplicar el análisis factorial.

La medida de adecuación de muestreo KMO. En nuestro caso, la medida de adecuación de muestreo KMO estuvo entre los coeficientes 1 y 0,8 catalogados como 'maravillosos', y 'meritorios' respectivamente. (García, 2001). Esto implica que las correlaciones entre pares de variables pueden explicarse por otras variables y que por lo tanto resulta adecuado la aplicación del análisis factorial.

Determinación de las comunalidades: Ios valores que aparecen en la columna de extracción se alejan de cero lo que nos indicó que la variable quedaba totalmente explicada por su componente al acercarse a un valor de uno.

Método de extracción: Para cada una de las cuatro dimensiones, se explicaron la: Varianza total, el gráfico de sedimentación y la Matriz de componentes, a través de los cuales, se corroboró que los ítems de cada apartado se podían resumir, o sea, se podían agrupar en componentes que explicaran la mayoría de la varianza observada en las respuestas ofrecidas por los encuestados, lo que implica que todas las variables estarían representadas de forma coherente.

Método de rotación: El procedimiento utilizado fue una rotación ortogonal Varimax para cada uno de los apartados, al tener, estas, un índice de correlación interfactores bajo, queda claro, que los componentes están relacionados entre sí.

Estos resultados, nos permitieron realizar un estudio confirmatorio, para cada dimensión y establecer que los ítems se ajustan a cada uno de los indicadores, lo que demuestra que existe consistencia interna entre los ítems del instrumento.

Fiabilidad del instrumento: Para obtener la fiabilidad de nuestro instrumento, se aplicó el Alpha de Crombach,

Tabla 2: Fiabilidad por Alpha de Cronbach

N of Cases $=119,0 \quad$ N of Items $=65$
Alpha $=0,9688$

tanto para el cuestionario completo como para cada uno de los apartados o dimensiones seleccionadas para conformar el mismo. Se administró el cuestionario a 119 estudiantes de segundo año, los cuales culminan la asignatura Inglés IV en el segundo semestre de este curso académico. (Manzano, 2007)

Como se puede observar en la tabla, los resultados obtenidos muestran un coeficiente de Alpha de Crombach de altísima fiabilidad.

\section{Conclusiones}

Con la construcción y validación de este cuestionario de evaluación de estrategias de lectura se logra que se concientice que el proceso de lectura va más allá del texto mismo, que el texto aporta significado pero el lector lo construye desde su propio conocimiento, experiencia personal y cosmovisión.

La lectura indudablemente es esencial durante el proceso de enseñanza-aprendizaje porque en ella intervienen procesos mentales de alto nivel, desarrolla destrezas y capacidades y posee potencialidades para ser explorada desde diferentes direcciones, sobre todo, los educadores de las diferentes ramas del saber, debemos tener conciencia; que la lectura está presente cotidianamente en la docencia, y que por lo tanto, es un problema que urge a todos perfeccionar desde nuestras propias perspectivas y posibilidades. De ahí que un trabajo consciente, interdisciplinar, sistemático, cohesionado y armónico con la comprensión, permitiría que el alumnado se apropie de algoritmos imprescindibles para el procesamiento de la información, como vía de solución para contribuir a la independencia cognoscitiva. (Manzano, et al. 2005)

El dominio de destrezas y desarrollo de capacidades en la lectura, contribuye al logro de la autonomía del estudiante durante la adquisición de los conocimientos en una lengua extranjera. Para lograr esas destrezas y capacidades, es necesario un trabajo consciente por parte del aprendiz y, también, la reflexión colectiva, en el avance personal de las estrategias a diferentes niveles de comprensión, para que éstas se desarrollen de manera integral.

El instrumento que se presenta, permite su utilización por apartados, en correspondencia con los objetivos que persigamos en nuestra práctica pedagógica, posibilita, además, que los sujetos del proceso de enseñanza-aprendizaje intercambien ideas, utilicen el proceso de lectura como medio y método de enseñanza y aprendizaje de forma eficaz y eficiente, lo que significa, construir significados y 
aplicarlos sistemáticamente para adquirir rapidez e independencia en el manejo de la información.

"Los medios pueden favorecer la actividad sujeto-sujeto y la interacción sujeto-sujeto, cuando representan un eslabón de enlace con el acervo cultural con el que el proceso de enseñanza-aprendizaje ha de pertrechar a los estudiantes" (Zilberstein y Collazo, 2009). El cuestionario de evaluación de lectura está en función de la actividad cognoscitiva y afectiva por lo que cumple varias funciones:

- Instructiva porque promueve la apropiación de los conocimientos, el desarrollo de habilidades, permite estudiar los objetos, fenómenos o procesos lo más objetivamente posible.

- Cibernética, ya que influye en las respuestas del estudiante, regula el proceso de enseñanza-aprendizaje, permite conocer las preferencias del estudiante, su motivación y manera de actuar.

- Formativa, pues influye en su formación, convicciones, valores, eleva su cultura, su cosmovisión y conocimiento de sí mismo.

- Recreativa, ya que favorece la distracción, permite el cambio de actividad, favorece la utilización de juegos.

- Desarrolladora, al favorecer la integralidad de la personalidad del estudiante.

- De control, pues propicia la observación, el autocontrol y la valoración del aprendizaje.

- Motivadora, innovadora y creadora, ya que motiva y entusiasma por la apropiación del contenido y despierta intereses e inclinaciones hacia la actividad creadora.

Desde el punto de vista práctico-metodológico; el cuestionario presentado, propone estrategias que pueden ser objetivos que propicien tareas o actividades relacionadas con los textos que se imparten durante la enseñanza y el aprendizaje de la lengua. Estas actividades posibilitan: la construcción de significados y sentidos, el desarrollo crítico y creativo del pensamiento a través del procesamiento de la información y alejan al proceso enseñanza-aprendizaje de actividades mecánicas y reproductivas, al permitir al estudiante que:

1. Activen sus conocimientos previos sobre el tema.

2. Tengan conciencia del objetivo de la lectura y la tarea que van a realizar.

3. Trabajen los tres significados que aporta un texto: el literal o explícito, el intencional o implícito y el cultural o complementario.
4. Desarrollen procesos de análisis - síntesis

5. Reflexionen sobre sus propias respuestas, para que conozcan sus procesos mentales y puedan utilizar estrategias de autocorrección.

6. Tengan en cuenta sus opiniones.

7. Reconozcan la utilidad de lo leído.

8. Reflexionen sobre situaciones problemáticas relacionadas con el tema.

9. Se motiven por la lectura de otros textos escritos sobre el tema.

10. Tomen conciencia de los niveles de ayuda que necesitan.

\section{Referencias bibliográficas}

- Ascuy, A. B. et al. (2007). Estrategia para el Perfeccionamiento de la Expresión Oral y la Escritura en Lenguas Extranjeras. Proyecto CITMA. Informe Parcial. UNICA, Ciego de Ávila.

- Brodie, P. e Irving, K. (2007). Assessment in work-based learning: investigating a pedagogical approach to enhance student learning. Assessment \& Evaluation in Higher education, 32 (1), 11-19.

- Fitzpatrick, (2006). An evaluative case study of the dilemmas experienced in designing a self-assessment strategy for Community Nursing students. Assessment \& Evaluation in Higher Education, 31 (1), 37-53.

- García, B. (2001). La cultura de la evaluación como proceso de mejora en un centro educativo. Tesis doctoral no publicada. Granada: Universidad de Granada.

- García J. E., Gil, J. y Rodríguez, G. (2000). Análisis Factorial. Madrid: La Muralla S. A.

- Gil, J. y Padilla, M. T. (2009). La participación del alumnado universitario en la evaluación del aprendizaje. Revista Educación XXI, (12), 43-65.

- González, M. (2000). Evaluación del aprendizaje en la enseñanza universitaria. Revista Pedagogía Universitaria. 5 (2) 1-121. Disponible en: http://www.uclm.es/profesorado/ricardo/docencia_e_investigacion/4/evaluacion_ halcones.doc [consulta 2006, 17de diciembre]

- González, M. (2009). La Evaluación del Aprendizaje. En fundamentos didácticos de la educación superior cubana. Selección de lecturas. La Habana: Félix Varela

\section{Cumbres}


- González, R. G. (2009).La clase de legua extranjera. Teoría y práctica. La Habana: Editorial Pueblo y Educación.

- Hidalgo, E. (1996). La evaluación en la ESO en el nuevo Sistema Educativo en Andalucía. Rev. RIE. Vol 14, n² 2, 51-73. Murcia.

- Hidalgo, E. et al. (2007): Formación en Competencias de Ciudadanía Europea. Granada. Edit. Método.

- Hidalgo, V. (2003). La mejora de un centro multicultural: de la ciudad autónoma de Ceuta. Tesis doctoral. Granada: Universidad de Granada.

- Leontiev, A. (1982). Actividad. Conciencia. Personalidad. La Habana: Editorial Pueblo y Educación.

- Maciel M. A. (2005). Comunicación Educativa: Dispositivo Holístico, Autorreferencial y de Duda, en la Construcción de Nuevas Competencias para la Formación y Desarrollo de lo Humano en la era Posmoderna. Razón y Palabra, 36, (Dic-Ene). Disponible en: http://www.cem. itesm.mx/dacs/publicaciones/logos/anteriores/n36/ onajera.html [consulta 2007, 27 de enero]

- Manzano, M. et al. (2005). La lectura - manantial de potencialidades. Revista Iberoamericana de Educación, 36, (9). Disponible en: http://www.rieoie.org/boletin36_9. htm

- Manzano, M. (2007). Estilos de aprendizaje, Estrategias de lectura y su relación con el rendimiento académico del inglés como lengua extranjera. Tesis del programa de Doctorado "Aportaciones Educativas en Ciencias Sociales y Humanas". Universidad de Granada-Universidad de Ciego de Ávila.

- Milán, R., Fuentes, H. C., De la Peña, R. (2009). La Evaluación como un proceso participativo. En fundamentos didácticos de la educación superior cubana. Selección de lecturas. La Habana: Félix Varela

- Zilberstein, J. y Collazo, R. (2009). Los medios de enseñanza-aprendizaje. En fundamentos didácticos de la educación superior cubana. Selección de lecturas. La Habana: Félix Varela 